\title{
Real-Time Soft-Finger Grasping of Physically Based Quasi-Rigid Objects
}

\author{
Maurizio de Pascale Gabriele Sarcuni Domenico Prattichizzo \\ DII - University of Siena, Italy \\ E-mail: [mdepascale, sarcuni, prattichizzo]@dii.unisi.it
}

\begin{abstract}
This paper describes the implementation of a demo that will be shown at the conference site. The demo of "softfinger grasping of physically based quasi-rigid objects" will provide solutions to grasp objects that are locally deformable and move according to rigid-body dynamics. This work summarizes the choices of the overall software architecture and the single algorithms used to run the simulation of "soft-finger grasping" in real time on high-end hardware. The soft-finger grasping is obtained extending a local model to include rotational friction while the local deformations are achieved through displacement fields directly on the GPU. Dynamics of the objects in the scene is simulated by a rigid body simulator which allows realistic interactions with other objects existing in the scene.
\end{abstract}

\section{Introduction}

In the last few years, the haptic science and technology allowed to achieve an unprecedented level of realism in user interaction with virtual environments. Even if current state of the art for multi-contact point interactions is not yet satisfactory, the use of more than one single-contactpoint devices (such as the PHANToM) has been shown to be quite satisfactory to handle 2 or 3 points of contact.

Deformable models for real time simulation is another key-topic, both for the graphic and the haptic research communities. Unfortunately the requirements needed for haptic rendering (i.e. higher rates, physical based behaviour) are more demanding and difficult to satisfy than those of graphic rendering. Methods and algorithms fitting the specific requirements of haptic rendering have been developed, ranging over different levels of realism and computational burden. Comparing the various approaches, advantages and disadvantages could be highlighted but so far no one has proved to be the ultimate solution to the ongoing issues.

Finally, the simulation of multi rigid objects, despite being a thoroughly developed subject, is yet computationally too heavy for use with complex scenes. The prohibitive Conference and Symposium 0-7695-2310-2/05 \$20.00 @ 2005 IEEE cost of computation involved in collision detection and resolution allows to simulate at haptic rates no more than few basic primitives (cubes and spheres).

Each one of these points has been addressed separately in the past, especially for deformable models which continues to be one of the main interesting research topics. The objective of this work is to show that current techniques can be integrated to achieve a high level of visuo-haptic realism at real time rates on high end personal computers.

\section{The Soft Finger Grasping Simulator}

To implement the proposed "Soft Grasping Simulator" three main techniques have been exploited and integrated: soft-finger grasping of rigid objects, local deformations for quasi-rigid objects and rigid body dynamics simulation.

\subsection{Soft Finger Grasping}

The type of grasp we are interested to simulate is the pinch grasp, in which the object is seized between two fingertips.

In such a grasp, the two contact forces splits into two components: the self-balanced grip forces and the load forces. The criteria for a stable pinch grasp is as follows: the sum of the load forces must be equal to the object's weight, the sum of the grip forces must be equal to zero and the contacts must not be slippery.

The grip forces are directly computed from the normal reaction applied on the haptic interfaces by the force rendering algorithm at the contact points. To obtain the load force a non-linear local model has been extended to include frictions. Several models for tangential friction are available in the literature among which we selected the model proposed in [6] which has proved to be simple and effective. One of the essential features of the human fingertips is the ability to resist moments about contact normals. If this ability is not present, even if the object could still be lifted up, it would continuously rotate about the line joining the two contact points. This is the reason why to implement a virtual grasp with two contact points it is necessary to include rotational friction to resist such torsional moments. 
For soft-finger we simply adapted the Zilles algorithm as in [5], taking into account angles of rotation around the contact normal if there is an interaction with only one point or otherwise about the line joining the two contact points if the interaction is with two contact points.

\subsection{Local Deformations}

Simulating deformable objects introduces relevant difficulties. The relative displacements of the object mass changes continuously thus modifying both the inertia tensor and the position of the mass center. Regarding collision detection and resolution, the pre-computed structures (such as the hierarchies of bounding primitives) become unusable or at least less efficient, given the need for continuous update to track the object deformations. Finally, computing the shape of deformation and the involved reaction forces introduce new and interesting problems, where the realism of the haptic rendering plays a crucial role.

Our work deals with simulation of quasi-rigid objects undergoing only local deformations, that is objects where the area interested in the deformation is small with respect to the whole object size. In these cases many of the fulldeformable issues can be disregarded: the inertia tensor of the object can be generally considered constant or can be quickly updated. The pre-computed structures for collisions detection can be used unchanged and simply coupled with a dynamic structure to handle contacts only in the few deformed regions.

In our demonstrative implementation, originally developed for rigid bodies, we have chosen to add deformations through the use of a GPU-friendly method [2] so that the added overhead for the CPU was negligible. The intrinsic parallelism of this model, where the dynamic behaviour of each vertex is independent from the others, allows for simulating deformations directly on the GPU. Moreover on GPUs supporting Shader Model 3.0 (i.e. vertex textures) almost all of the computational burden can be removed from the CPU.

\subsection{Rigid body dynamics}

To simulate rigid body dynamics, we initially used ODE [8], an open source dynamic engine, used by many research communities and some commercial projects. Unfortunately ODE proved to be a not yet mature product, so we moved to the Novodex SDK [7], a free, for noncommercial use, dynamic engine used especially in the game development industry. After tests and experiments, we ended up with an hybrid approach using mainly custom code for both collision and integration of dynamics, which proved to be fastest and more reliable than counterparts, and using the third party physic engine only when almostresting contacts (which are the more complex issues) were detected. Objects in haptic device islands (i.e. influencroceedings of the First Joint Eurohaptics Conference and Symposium on Haptic Interfaces for Virtual Environment and Teleoperator Systems 0-7695-2310-2/05 \$20.00 @ 2005 IEEE ing a device) were simulated with a simple Euler solver running in the haptic thread while for the others a RungeKutta based solver, running in the graphic thread, was used. Dynamics from the two threads were blended based on the distance from haptic devices, with the fast one driving the slow one. For the broad collision phase an interruptible hierarchies of bounding spheres [4] was used, while for the narrow phase standard triangle-triangle collision tests, but taking triangle normals into account, were used.

\section{Conclusions}

The objective of this work was to show how current hardware and technologies can be used to implement a grasping simulator able to run at real time rates with high visuo-haptic realism. The demo has been tested using a Phantom Desktop and a Phantom Premium 1.5, as shown in Fig. 1, running on a 2 Ghz Pentium 4 equipped with a NVIDIA GeForce FX 5600. The performance of the overall demo was satisfactory and with a good level of realism for the pinch grasp. The most up to date implementation will be show at the conference during the demo sessions.

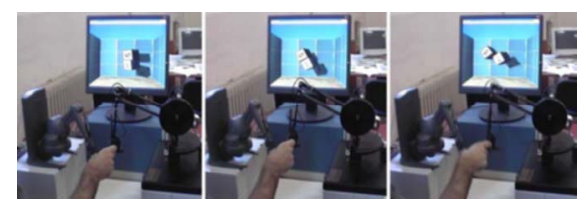

Figure 1: Screenshots from physically based interaction

\section{References}

[1] Baraff, D., Witkin, A., "Dynamic Simulation of Non-penetrating Flexible Bodies", SIGGRAPH 1992 Conference Proceedings, 1992, pp. 303-308.

[2] de Pascale, M., de Pascale, G., Prattichizzo, D., Barbagli, F., "A GPU-friendly Method for Haptic and Graphic Rendering of Deformable Objects", Proceedings of Eurohaptics 2004, 2004, pp. 44-51.

[3] Johansson, R.S., Westling, G., "Factors influencing the force control during precision grip", Experimental Brain Research, 1998, pp. 53:277284.

[4] O’Sullivan, C., Dingliana, J., "Graceful Degradation of collision handling in physically based animation", Proc. Eurographics 2000, 2000, pp. 239-247.

[5] Salisbury, K., Barbagli, F., Frisoli, A., Bergamasco, M., "Simulating human fingers: a Soft Finger Proxy Model and Algorithm", Haptic Symposium 2004, 2004, pp. 9-17 (Vol.1).

[6] Zilles, C.B., "Haptic Rendering with the Toolhandle Haptic Interface”, MIT Department of Mechanical Engineering, Thesis, 1995.

[7] http://www.novodex.com

[8] http://ode.org 